\title{
Oscillatory and Steady Flows in the Annular Fluid Layer inside a Rotating Cylinder
}

\author{
Veronika Dyakova and Denis Polezhaev \\ Laboratory of Vibrational Hydromechanics, Perm State Humanitarian Pedagogical University, 24 Sibirskaya Street, \\ Perm 614990, Russia \\ Correspondence should be addressed to Denis Polezhaev; polezhaev@pspu.ru
}

Received 6 June 2015; Accepted 1 November 2015

Academic Editor: Matteo Aureli

Copyright (C) 2016 V. Dyakova and D. Polezhaev. This is an open access article distributed under the Creative Commons Attribution License, which permits unrestricted use, distribution, and reproduction in any medium, provided the original work is properly cited.

The dynamics of a low-viscosity fluid inside a rapidly rotating horizontal cylinder were experimentally studied. In the rotating frame, the force of gravity induces azimuthal fluid oscillations at a frequency equal to the velocity of the cylinder's rotation. This flow is responsible for a series of phenomena, such as the onset of centrifugal instability in the Stokes layer and the growth of the relief at the interface between the fluid and the granular medium inside the rotating cylinder. The phase inhomogeneity of the oscillatory fluid flow in the viscous boundary layers near the rigid wall and the free surface generates the azimuthal steady streaming. We studied the relative contribution of the viscous boundary layers in the generation of the steady streaming. It is revealed that the velocity of the steady streaming can be calculated using the velocity of the oscillatory fluid motion.

\section{Introduction}

The rotational and oscillatory flows of a fluid with a free surface in a container are of fundamental interest and are interesting for certain applications. When the horizontal cylinder is stationary, the liquid is at rest in a pool at the bottom of the cylinder. Inside a moving cylinder, the fluid may move. Practical examples consist of undesirable slosh in spacecraft storage tanks and in aircrafts, rockets, road, and marine vehicles transporting liquids (for a brief review, see [1]). When the cylinder rotates with a low to moderate angular velocity, its rising side drags out a thin film of liquid from the pool. This flow regime has been studied in detail, and an entire series of instabilities has been discovered including hygrocysts, solitary waves, and shark-teeth and fish-like patterns [2].

We experimentally examined the dynamics of an annular layer of a low-viscosity liquid inside a rapidly rotating horizontal cylinder. Inside a rapidly rotating cylinder, the fluid is observed to be almost in rigid body rotation. The force of gravity prevents the rise of the fluid on the ascending wall of the rotating cylinder, so the angular velocity of the fluid $\Omega_{l}$ is less than the velocity of the rotation of the cylinder $\Omega$. Conversely, the force of gravity draws the liquid down near the descending wall such that $\Omega_{l}>\Omega$. The continuity of the fluid requires the annular layer to be thinner near the descending wall with the minimum thickness at the bottom of the rotating cylinder (Figure 1). The free surface of an annular fluid layer is stationary in the laboratory frame and has a form of a cylinder whose axis is below the axis of the rotating cylinder. In the rotating frame, the fluid distribution is considered to be a two-dimensional surface wave propagating in the direction opposite to the cylinder's rotation.

Phillips [3] theoretically showed that the amplitude of the displacement of the interface between the liquid and air $A$ is proportional to the parameter $\Gamma \equiv g / \Omega^{2} a$, where $g$ is the gravitational acceleration and $a$ is the radius of the free surface of the fluid. For certain combinations of the rotation rate and the liquid volume, a resonant excitation of the surface waves with various axial and azimuthal wavenumbers was demonstrated.

The oscillatory motion of the annular layer is of great interest for both fundamental hydrodynamic research and astrophysical applications. Dyakova et al. [4] experimentally determined that the oscillatory fluid flow above the granular medium inside a rotating cylinder causes the onset of the instability at the fluid-granular medium interface and that 


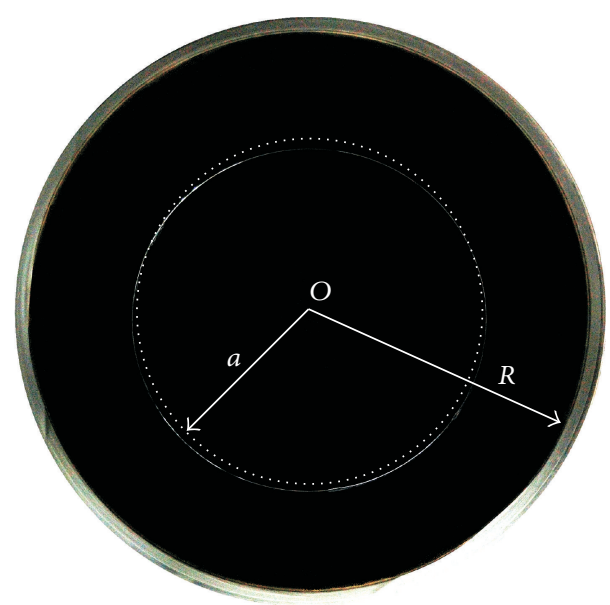

FIGURE 1: The annular layer of PMS-20 silicon oil inside the cylinder with radius $R=6.3 \mathrm{~cm}$ (rotation rate $f=5 \mathrm{rps}$, relative filling $q=0.61$ ): the solid line shows the interface between the fluid and the air; the interface is displaced from the equilibrium position with the centre at $O$ (dotted line); $a$ is the radius of the air column. The cylinder rotates anticlockwise.

the subsequent onset of a quasistationary relief in the form of ripples extended along the axis of rotation. The stability parameter requires determination of the velocity of the fluid oscillations near the interface. Here, we present the initial experimental results on the velocity of the oscillatory fluid flow inside a rapidly rotating cylinder. We expect that the data obtained will facilitate the analysis of the phenomenon observed by Dyakova et al. [4].

Furthermore, oscillatory fluid motion is centrifugally unstable to the onset of spatially periodic flow similar to Taylor-Gortler vortices [5]. The dynamics of an annular fluid layer inside a rotating cylinder in the field of gravity is qualitatively similar to libration-driven flows. Many librating astrophysical bodies are believed to possess an internal fluid layer either in the form of a subsurface ocean or in the form of a partially molten iron core $[6,7]$. Despite the large number of phenomena caused by the oscillatory flow in an annular fluid layer, the intensity of this flow has not been studied experimentally.

The two-dimensional oscillatory flow in an annular fluid layer is a source of azimuthal steady streaming. The progressive azimuthal wave produces boundary layers at both the bottom and the free surface. Batchelor [8] demonstrated that a progressive wave generates a mean vorticity in the Stokes boundary layer near the bottom such that the fluid elements just outside the boundary layer drift in the direction of the wave propagation. In accordance with [8], the drift (masstransport) velocity is independent of the viscosity and has the value

$$
u_{\mathrm{ss}}=\frac{5}{4} \frac{U_{\mathrm{osc}}^{2}}{u_{\mathrm{wave}}},
$$

where $U_{\text {osc }}$ is the velocity amplitude of the periodic motion and $u_{\text {wave }}$ is the phase velocity of the surface wave. As noted by Longuet-Higgins [9], just below the boundary layer at the free surface the vertical gradient of the second-order drift velocity is given by

$$
\frac{\partial u_{\mathrm{fs}}}{\partial z}=4 A^{2} \Omega_{\mathrm{osc}} k^{2} \operatorname{coth} k h,
$$

where $A$ denotes the amplitude of the wave at the surface, $\Omega_{\text {osc }}$ is the radian frequency of the fluid oscillations, $k$ is the wavenumber, $h$ is the thickness of the fluid layer, and coordinate $z$ is measured vertically downwards.

The additional steady streaming could be an effect of the chemical heterogeneity of the fluid and the subsequent increase of the surface tension gradient on the free surface. This flow is referred to as Marangoni solutocapillary flow and is directed along the gradient of the surface tension [10]. The addition of surfactant to the fluid reduces the free surface energy of the fluid and changes the boundary conditions for the velocity at the free surface [11]. In the limiting case with a high concentration of the surfactant, the free surface becomes solid-like and the solutocapillary flow vanishes. The replacement of the free surface of an annular layer of pure fluid inside a rotating cylinder with a solid surface causes the solid surface to generate azimuthal steady streaming. In accordance with [12], the velocity of this flow is much greater than the values obtained from (1) and (2).

The other source of the intensification of the azimuthal steady streaming is the resonant excitation of progressive and standing surface waves in the annular layer of the fluid [3]. Ivanova et al. [13] experimentally discovered the existence of the azimuthal steady streaming and studied the influence of centrifugal waves on this flow.

For the first time, we report experimental data regarding the velocity of the oscillatory flow and the steady streaming in the annular fluid layer inside a rapidly rotating cylinder. The experiments were conducted with fluids without surfactants and in the absence of surface waves, which implies that the steady streaming was generated only by the viscous boundary layers at the rigid bottom and at the free surface. The results of the analysis allow us to assess the contribution of these sources to the velocity of the azimuthal steady streaming.

\section{Experimental Setup}

The experimental setup is shown in Figure 2. The transparent circular cylinder 1 of length $L=7.8 \mathrm{~cm}$ and diameter $D=$ $12.6 \mathrm{~cm}$ was fitted horizontally into ball bearings that were mounted onto rigid stand 2 . The stepper motor 3 was coupled to the cylinder and provided rotation in the range from 0 to $70 \mathrm{~s}^{-1}$ with an accuracy of $0.05 \%$.

The experiments were conducted using silicon oil PMS20 with a viscosity of $20 \mathrm{cSt}$ and a density of $\rho_{l}=0.87 \mathrm{~g} / \mathrm{cm}^{3}$. The quantity of the liquid is characterized by the relative filling $q=V / V_{0}$ ( $V$ is the fluid volume; $V_{0}$ is the cavity volume), which varied in the range from 0.1 to 0.6 . We used Styrofoam markers with a size of $1 \mathrm{~mm}$ and a density of $\rho \approx$ $0.02 \mathrm{~g} / \mathrm{cm}^{3}$ to measure the free-surface velocity. The markers on the free surface were illuminated using stroboscopic light 4 , which had frequency equal to the cylinder rotation rate. 


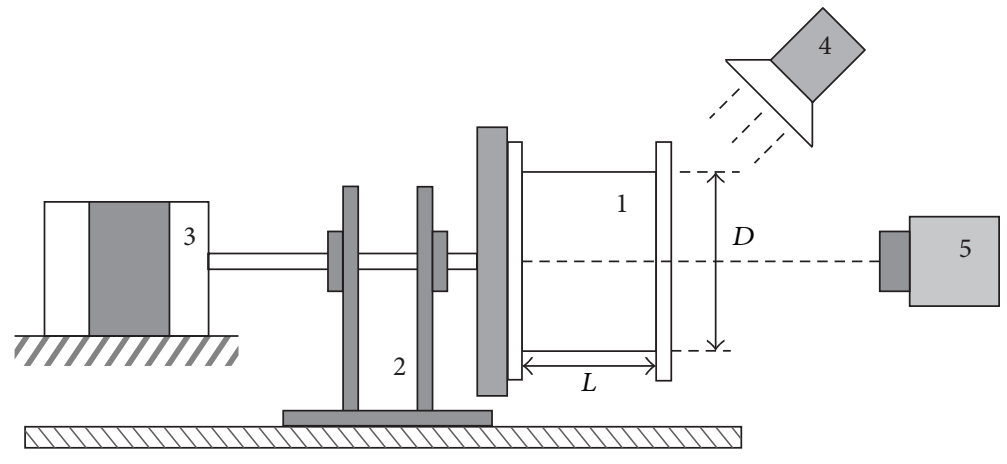

FIGURE 2: Sketch of experimental setup.

Images of the fluid were recorded using a high-speed camera 5, which was positioned at a right angle to the cylinder axis. The camera provided registration of the free surface with frame rates of up to $500 \mathrm{fps}$ at a resolution of $1280 \times 1024$ pixels.

Each experiment followed a similar protocol. When the cylinder was partially filled with fluid and rotated sufficiently rapidly, the fluid underwent nearly rigid-body rotation about a central air column. At the definite rotation rate, the masstransport free surface velocity was measured, and images of the free surface were recorded using the high-speed camera after several minutes had elapsed. The rotation rate was then decreased by $\Delta f=\Delta \Omega / 2 \pi=1 \mathrm{rps}$, and the procedure was repeated.

\section{Oscillatory Fluid Motion}

Inside a rapidly rotating cylinder, the annular layer thickness varies along the azimuthal coordinate: the layer has the greatest thickness at the upper pole and the least thickness at the bottom of the cylinder (Figure 1).

After centrifugation, the light markers occupied arbitrary positions at the free surface. According to the observations, the angular velocity of the markers (i.e., liquid) $\Omega_{l}$ was less than the velocity of the rotation of the cylinder $\Omega$, so the liquid performed retrograde azimuthal steady streaming.

Note that the steady streaming is two-dimensional. In addition to the azimuthal motion, axial drift was also present: markers at the free surface moved slowly to the nearest endwall. After sticking to the end-wall, the particles never came back to the central part of the cylinder. The origin of the axial drift will be discussed in detail in the "Azimuthal Steady Streaming" section. Here, we simply note that the period of the azimuthal steady streaming was approximately $10^{1}-$ $10^{2} \mathrm{~s}$, and the typical time of the axial drift was approximately $10^{2}-10^{3} \mathrm{~s}$. The azimuthal fluid motion was almost one-dimensional during the several cycles of the cavity rotation, which allows for the measurement of the azimuthal component of the fluid velocity.

Figure 3(a) illustrates the evolution of the marker's position at the free surface in the rotating frame. The angle $\varphi$ denotes the azimuthal position of the marker in the rotating frame. Figure 3(a) clearly reveals that the fluid undergoes harmonic oscillations as the amplitude remains constant at a fixed rotation rate $\Omega$ while the frequency of oscillations $\Omega_{\text {osc }}=\Omega$. In addition to the oscillatory motion in the azimuthal direction, a slow retrograde rotation of the fluid is also observed: the angle between the marker and the cylinder $\varphi$ decreases over time.

Here, we focus on the oscillatory fluid motion. Let us follow the evolution of the marker during the rotation cycle (Figure 3(b)). The angle $\theta$ denotes the angle of the cylinder rotation with respect to the vertical axis; $\theta=0$ is taken vertically downwards. The maximum displacement from the equilibrium position of the marker is observed at angles $\theta=$ $90^{\circ}+\pi n$, where $n=1,2,3, \ldots$. Figure $3(\mathrm{~b})$ shows that the oscillatory fluid motion obeys the law $\varphi=\varphi_{0} \sin \Omega t$ or $\varphi=$ $\varphi_{0} \sin \theta$. Thereafter, the velocity of the oscillatory motion is equal to $u_{\mathrm{osc}}=U_{\mathrm{osc}} \cos \theta$. This result favourably agrees with theoretical speculations: $u_{\text {osc }}$ is maximum where the layer thickness $h$ is minimum (at $\theta=0$ ) and $u_{\mathrm{osc}}$ is minimum at the upper pole of the cylinder (at $\theta=180^{\circ}$ ).

The dynamics of the annular flow of a low-viscosity fluid is governed by the interplay between the force of gravity and the centrifugal force. Following Ivanova et al. [13], we introduce the dimensionless parameter $\Gamma=g / \Omega^{2} a$ ( $g$ is the acceleration of gravity and $a$ is the radius of the cylindrical free surface), which is the ratio of the gravitational force to the centrifugal force. Inside a rapidly rotating cylinder, the effect of gravity is negligible; hence, the fluid rotates as a rigid body. In contrast, inside a slowly rotating cylinder, the effect of gravity becomes significant, and a collapse of the annular flow may occur.

Additionally, for certain combinations of the rotation rate and the liquid volume, the excitation of the surface waves with various axial and azimuthal wavenumbers is possible $[4,13]$. The surface wave generates an oscillatory fluid flow, which has a frequency that is determined by the phase velocity of the wave. Then, the fluid motion becomes more complicated and, therefore, cannot be regarded as a superposition of the forced oscillations and the azimuthal steady streaming (Figure 4(a)).

Fourier analysis shows that the oscillatory fluid motion is a superposition of two harmonic oscillations (Figure 4(b)). First, the forced oscillations have a frequency equal to the rotation rate of the cylinder. The second peak in Figure 4(b) corresponds to the wavy perturbations, which are excited 


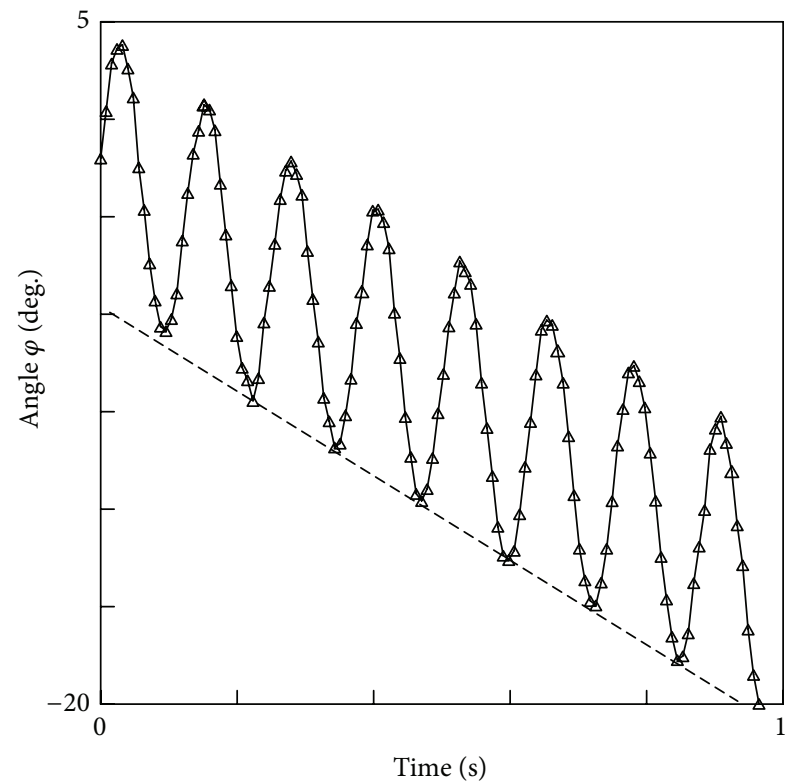

(a)

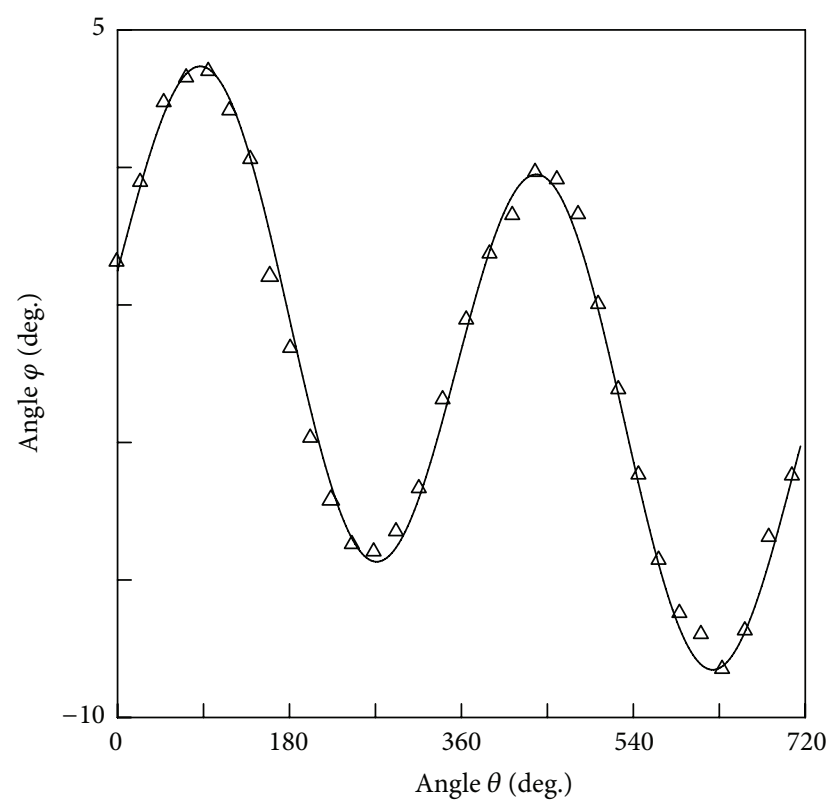

(b)

FIGURE 3: Time evolution of the azimuthal position of the marker at the free surface in the rotating frame; the dotted line indicates the azimuthal steady streaming (a); azimuthal position of the marker $\varphi$ versus the angle of the cylinder's rotation $\theta$ (b); $f=8 \mathrm{rps}, q=0.51$.

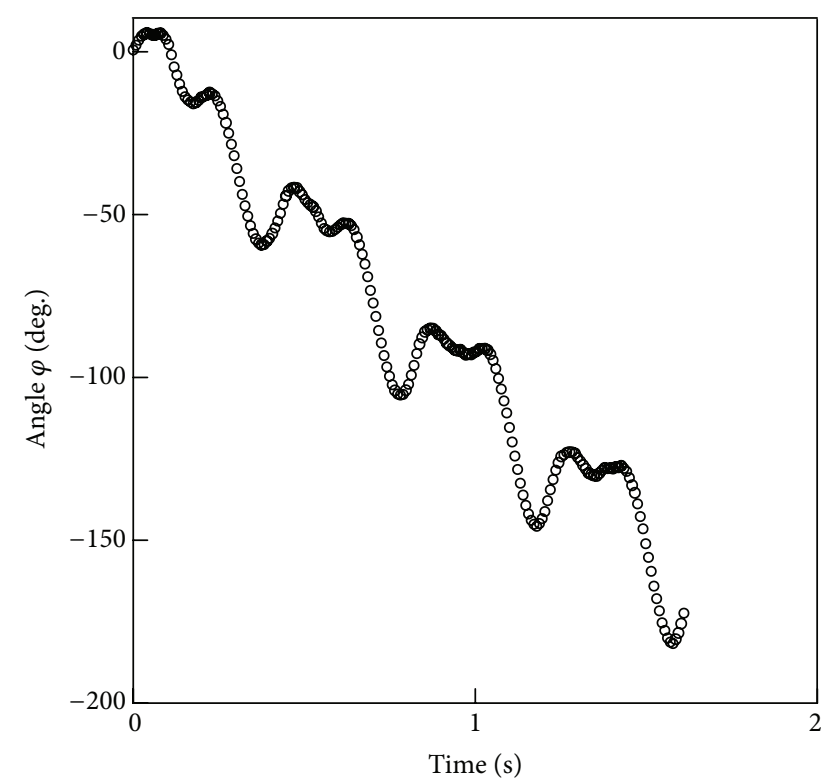

(a)

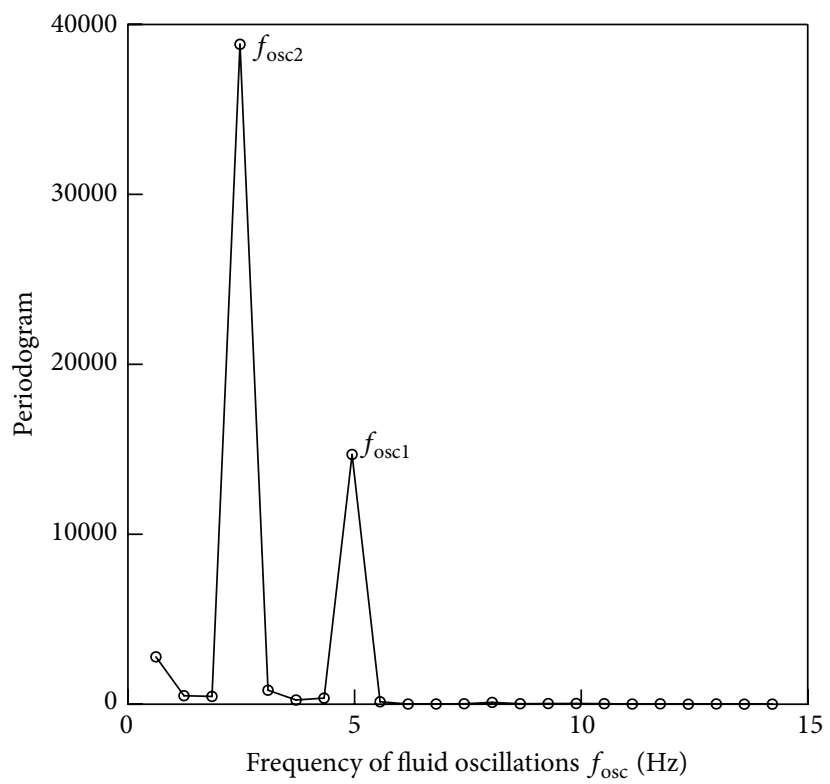

(b)

Figure 4: Time evolution of the azimuthal position of the marker at the free surface in the presence of a surface wave (a); the results of the Fourier analysis: $f_{\text {osc1 }} \approx 5 \mathrm{~Hz}, f_{\text {osc } 2} \approx 2.5 \mathrm{~Hz}(\mathrm{~b}) ; f=5.25 \mathrm{rps}, q=0.21$.

under the influence of the surface wave. The wave processes in the annular fluid layer were analysed in more detail in [14].

Hereafter, we will consider only forced fluid oscillations. Because the fluid oscillates under the force of gravity, the amplitude of the forced oscillations is determined by the dimensionless acceleration $\Gamma$. Indeed, an increase in the cylinder rotation rate (decrease in $\Gamma$ ) causes a decrease in the amplitude of the fluid oscillation (Figure 5(a)). Conversely, an increase in the relative filling $q$ (i.e., a decrease in the radius $a$ and an increase in $\Gamma$ ) is followed by an increase in the amplitude of the forced oscillations at a fixed rotation rate (Figure 5(b)).

The summarized results are shown in the plane of the dimensionless parameters $\Gamma$ and $U_{\text {osc }} / \Omega R$ (Figure 6). Note 


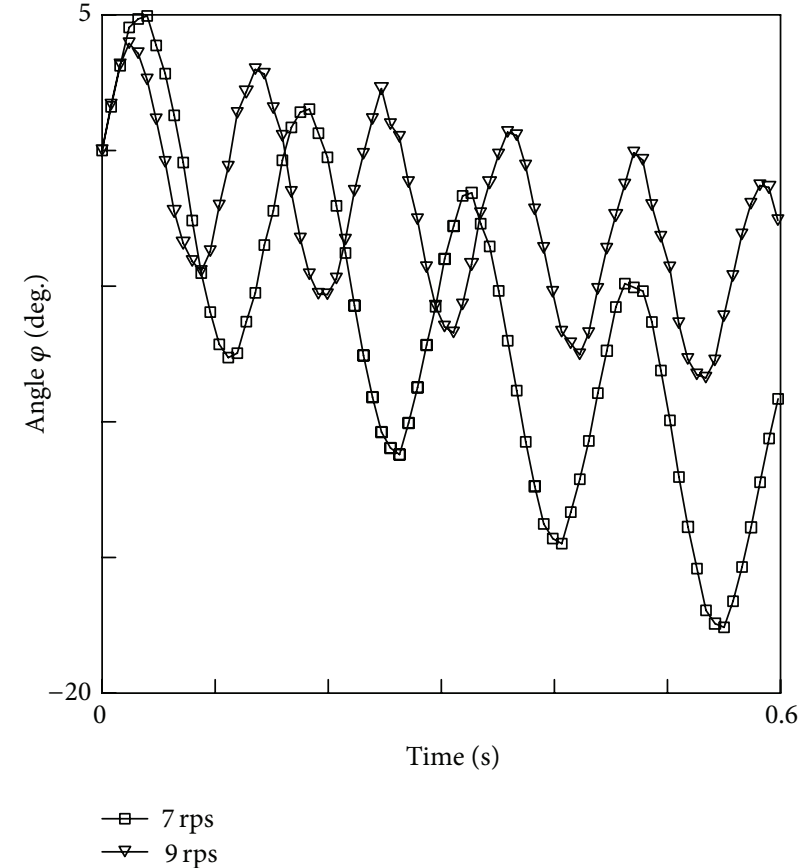

(a)

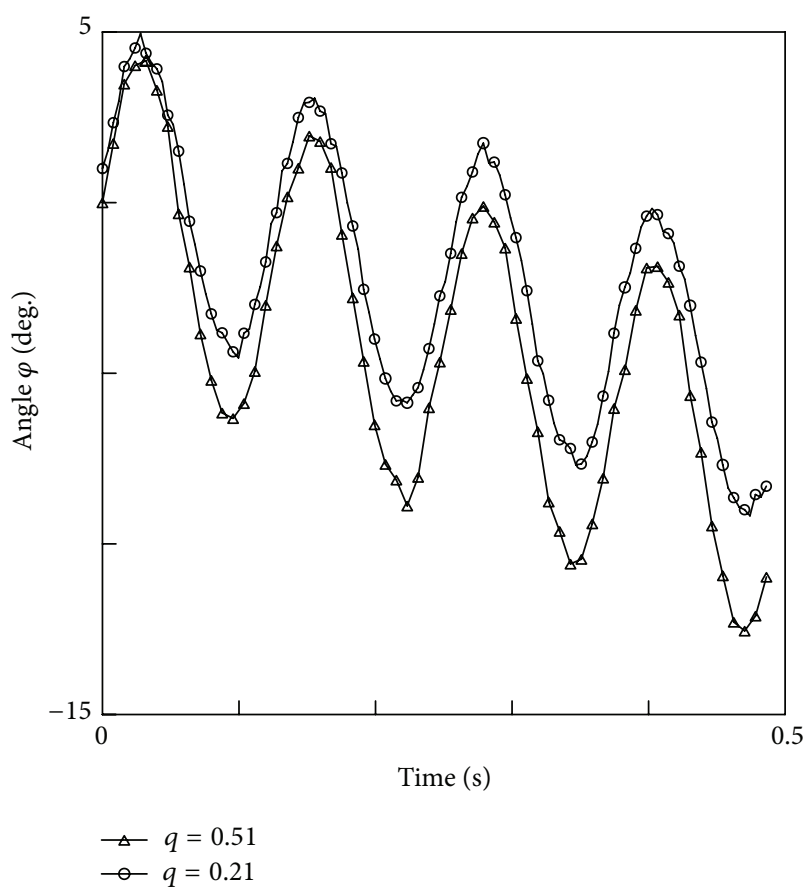

(b)

FIGURE 5: Time evolution of the azimuthal position of the marker at the free surface, rotation rate $f=7$ and 9 rps, relative filling $q=0.51$ (a); $f=8 \mathrm{rps}, q=0.21$ and 0.51 (b).

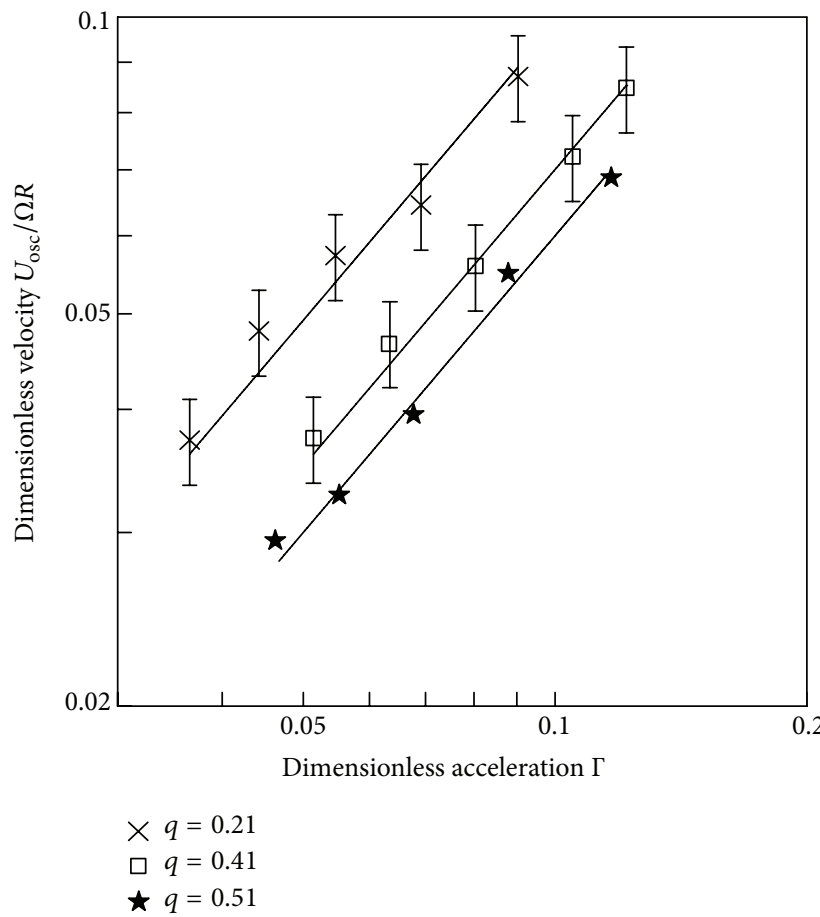

FIGURE 6: Dimensionless velocity of the oscillatory fluid flow versus the dimensionless acceleration at different values of the relative filling $q$.

that, regardless of the fluid volume, the velocity obeys the law $U_{\mathrm{osc}} / \Omega R \sim \Gamma$. These experimental results are in good agreement with each other in the plane of the dimensionless parameters $\Gamma c\left(1+c^{2}\right)$ and $U_{\text {osc }} / \Omega R$ (Figure 7), where $c \equiv$ $a / R=(1-q)^{1 / 2}$ is the dimensionless radius of the free surface. The experimental data agree well with the theoretical predictions made by Phillips [3] (dotted line in Figure 7):

$$
\frac{u_{\mathrm{osc}}}{\Omega R}=\frac{1}{2} \Gamma c\left(1+c^{2}\right) \cos \theta
$$




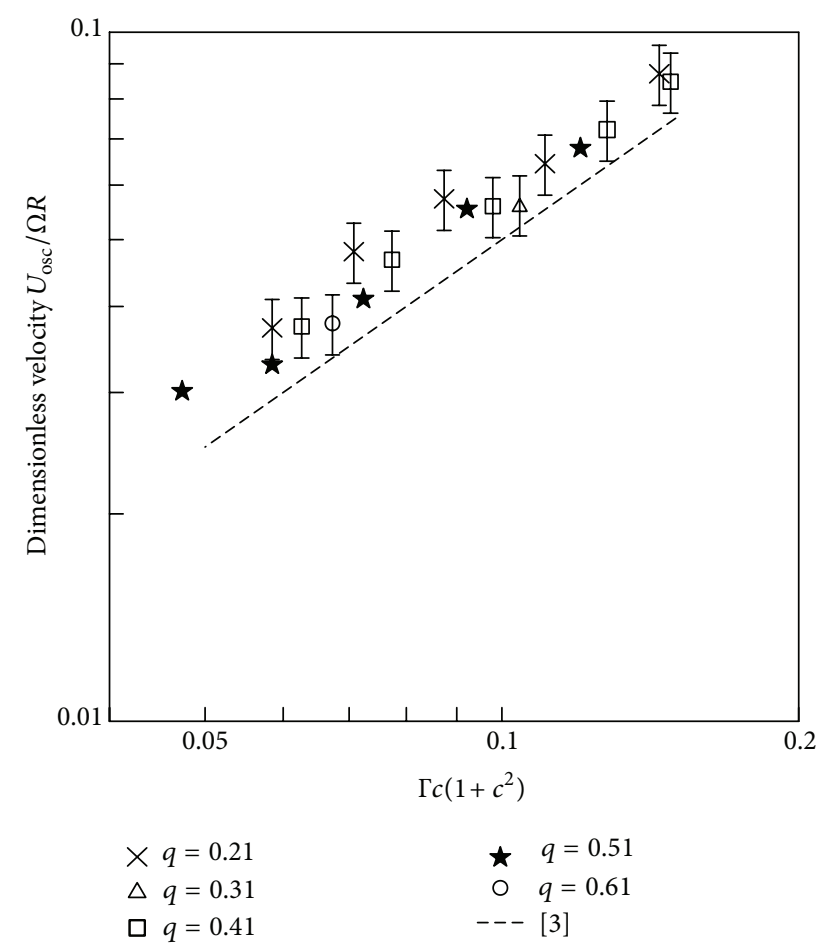

FIgURE 7: Dimensionless velocity of the oscillatory fluid flow $U_{\text {osc }} / \Omega R$ versus the dimensionless parameter $\Gamma c\left(1+c^{2}\right)$; the dotted line shows the theoretical results [3].

The mismatch between the theoretical and experimental results can be explained by the fact that (3) is obtained using the inviscid approximation. This condition is satisfied only if the layer thickness $h$ is much greater than the thickness of the viscous boundary layers at both the bottom and the free surface $\delta=(2 v / \Omega)^{1 / 2}$. In the experiments, the dimensionless layer thickness $\gamma \equiv h / \delta$ is relatively small and varies within the limits from 7 to 30 .

\section{Azimuthal Steady Streaming}

In addition to the forced oscillations, the steady flow of the annular fluid layer was observed. In the rotating frame, the fluid distribution is considered as a two-dimensional surface wave propagating in the direction opposite to the cylinder's rotation. The azimuth wave generates a mean vorticity in the viscous boundary layers at both the bottom and the free surface and gives rise to the azimuthal drift just outside the boundary layers in the direction of the wave propagation. The effect of the Stokes boundary layer near the rigid wall in the approximation of the thin annular layer was studied in detail by Ivanova et al. [13]. The effect of the boundary layer near the free surface of the fluid inside a rotating cylinder has not yet been studied.

According to the observations, the steady streaming was two-dimensional: the velocity of a fluid element had azimuthal and axial components, but the axial drift was much slower than the azimuthal steady flow. The azimuthal fluid drift was uniform along the axis of rotation except for

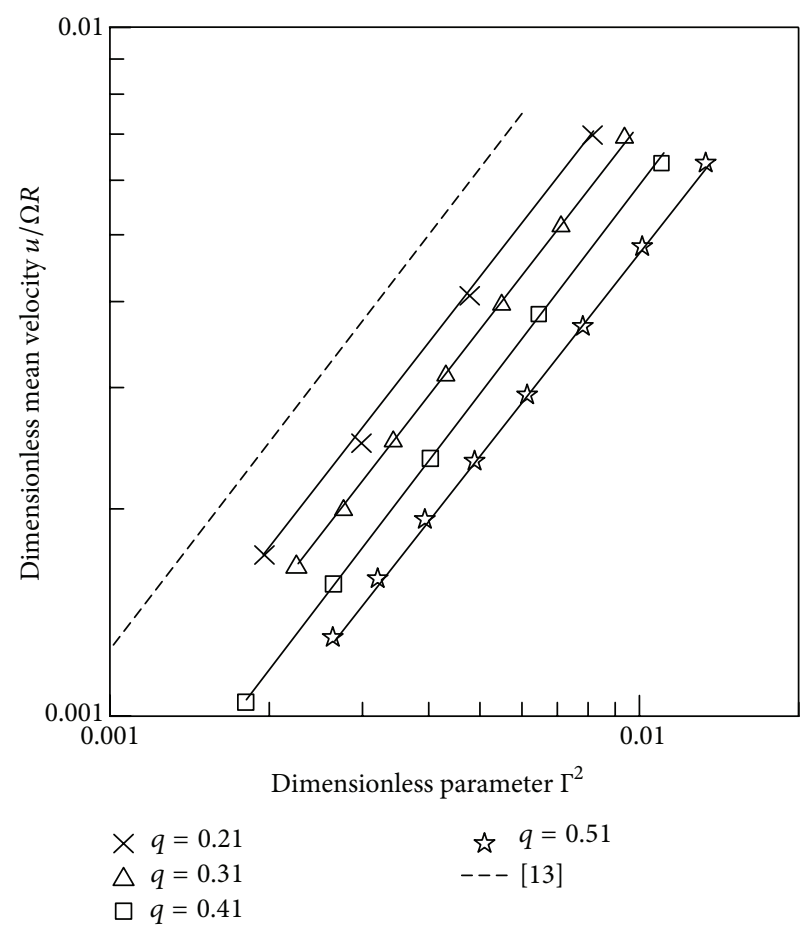

FIGURE 8: Dimensionless velocity of the azimuthal steady flow $u / \Omega R$ versus the dimensionless acceleration $\Gamma$; the dotted line shows the results obtained in the approximation of the thin annular layer [13].

the short distance near the end-walls of the cylinder: the fluid satisfies the no-slip condition at the end-walls. Therefore, the viscous boundary layer (Ekman layer) develops near the endwall. As previously mentioned, the fluid rotates slower than the cylinder (see, e.g., Figure 3(a)). Near the end-wall, the fluid element experiences a centrifugal force that is greater than the centrifugal force away from the end-wall. Due to the centrifugal force, the fluid close to the end-wall moves away from the centre and then travels along the cylindrical wall. Then, two fluid flows collide in a central part of the cylinder and turn inwards. Close to the free surface, the fluid flow returns to the rotating end-wall. Therefore, there is a two-vortex fluid flow directed towards the centre of the cylinder near the wall and toward the end-walls at the free surface. When the intensity of the axial drift is greater, the velocity of the azimuthal steady streaming is greater. The effect of the steady flow generated by the Ekman layer is a specific feature of rotating flows: this phenomenon is known as Ekman pumping [15].

It is worth noting that, if the fluid rotates faster than the cylinder and provokes the onset of centrifugal instability, then the axial inhomogeneity of the azimuthal rotation causes the appearance of spiral Taylor-Gortler vortices [16].

As the steady motion is induced by the forced oscillations, the intensity of the motion will depend on the parameter $\Gamma$ (Figure 8). Ivanova et al. [13] calculated the azimuthal drift velocity in an approximation of the thin annular layer $c \rightarrow 1$ :

$$
\frac{u}{\Omega R}=\frac{5}{4} \Gamma^{2}
$$


This result is shown by the dotted line in Figure 8. It is easily revealed from the figure that the experimental results tend to the predictions of Ivanova et al. [13] as long as the relative filling $q \rightarrow 0(c \rightarrow 1)$. Nevertheless, we still face the problem of finding a universal law that is valid for all values of $c$. To do this, we must first identify all the sources of the steady streaming.

As previously mentioned, the azimuthal steady streaming is generated in the viscous boundary layers at the rigid wall and near the free surface. It is important to determine the relative contribution of each generator to the velocity profile. Based on (1) and (2), we can make a qualitative assessment of the theoretical data from $[8,9]$. Our goal is to estimate the parameters $u_{\mathrm{ss}}$ and $u_{\mathrm{fs}}$ so that we can calculate the quantities in (1) and (2). In the rotating frame, a fluid distribution is considered to be a two-dimensional surface wave propagating in the direction opposite to the cylinder's rotation so that the phase velocity of the wave $u_{\text {wave }}=\Omega a$. The surface wave induces fluid oscillations and has a frequency that is equal to the rotation rate of the cylinder: $\Omega_{\mathrm{osc}}=\Omega$.

The velocity of the oscillatory flow can be found from the equation $U_{\text {osc }}=\varphi_{0} a \Omega\left(\varphi_{0}\right.$ is the angular oscillation amplitude). Thus, (1) takes the following form:

$$
u_{\mathrm{ss}}=\frac{5}{4} \frac{U_{\mathrm{osc}}^{2}}{u_{\mathrm{wave}}}=\frac{5}{4} \frac{\varphi_{0}^{2} a^{2} \Omega^{2}}{\Omega a}=\frac{5}{4} \varphi_{0}^{2} a \Omega .
$$

Equation (2) illustrates the vertical gradient of the masstransport velocity just below the boundary layer at the free surface. Assuming that the curvature of the layer is negligible and the gradient $\partial u_{\mathrm{fs}} / \partial z$ does not change with depth, we can estimate the velocity $u_{\mathrm{fs}}$ :

$$
u_{\mathrm{fs}}=4 A^{2} \Omega_{\mathrm{osc}} k^{2} h \operatorname{coth} k h,
$$

where $A$ denotes the amplitude of the wave at the free surface, $k \equiv 2 \pi / \lambda$ is the wavenumber, and $h$ is the thickness of the fluid layer. The amplitude is equal to the displacement of the free surface from the equilibrium position $A=(1 / 2)(1-$ $\left.a^{2} / R^{2}\right)\left(g / \Omega^{2}\right)$ [3], where $g$ is the gravitational acceleration. The wavelength $\lambda=2 \pi a$ such that $k h=h / a$.

The typical layer thickness $h \sim 10^{-2} \mathrm{~m}$ and $a=R-h \approx$ $5 \times 10^{-2} \mathrm{~m}$; hence, $k h=1 / 5$ and $\operatorname{coth} k h \approx 1 / k h$. Thus, (6) takes the following form:

$$
u_{\mathrm{fs}}=\left(1-\frac{a^{2}}{R^{2}}\right)^{2} \frac{g^{2}}{\Omega^{3} a} .
$$

Using (5) and (7), we can estimate the relative contribution of the two mechanisms:

$$
\frac{u_{\mathrm{fs}}}{u_{\mathrm{ss}}}=\frac{4}{5}\left(1-\frac{a^{2}}{R^{2}}\right)^{2} \frac{g^{2}}{\Omega^{4} a^{2} \varphi_{0}^{2}},
$$

where $\Omega \sim 50 \mathrm{rad} / \mathrm{s}, \varphi_{0} \sim 10^{-1} \mathrm{rad}$, and $g \approx 10 \mathrm{~m} / \mathrm{s}^{2}$. After substituting in the equation above, we obtain the formula

$$
\frac{u_{\mathrm{fs}}}{u_{\mathrm{ss}}} \sim 10^{-1} .
$$

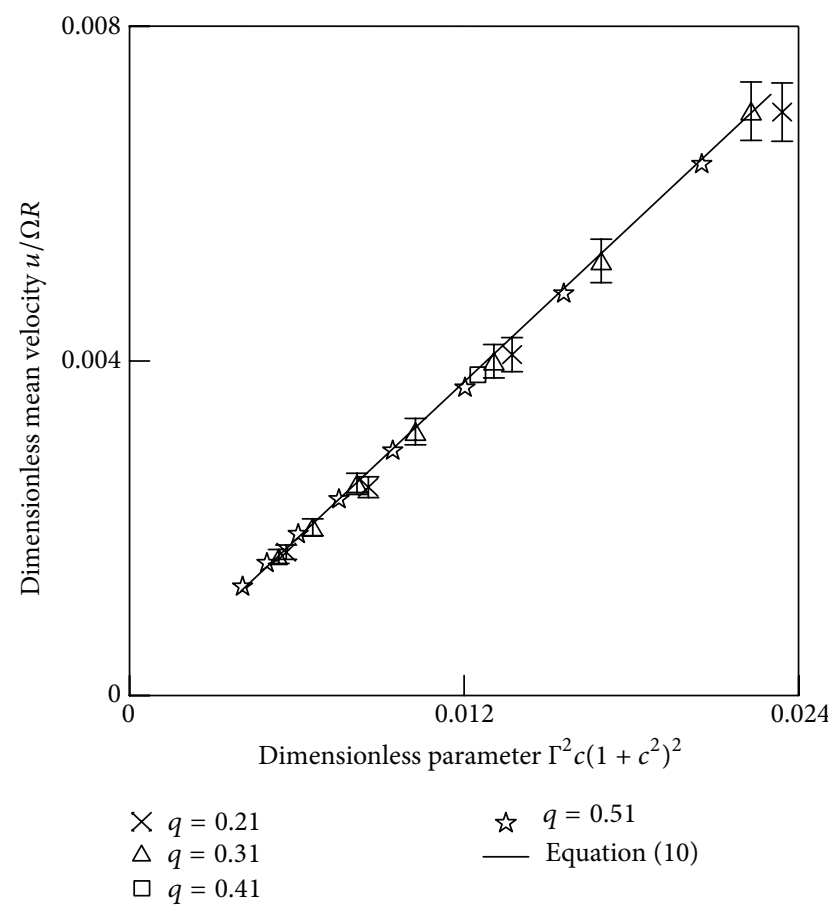

FIGURE 9: Dimensionless velocity of the steady flow $u / \Omega R$ versus the dimensionless parameter $\Gamma^{2} c\left(1+c^{2}\right)^{2}$; the solid line shows the results obtained from (10).

Formula (9) indicates that the azimuthal steady streaming is mainly generated in the Stokes boundary layer. The azimuthal velocity of the steady flow can be calculated from (1) and (3). Therefore,

$$
\frac{u}{\Omega R}=\frac{5}{4} \frac{U_{\mathrm{osc}}^{2}}{u_{\mathrm{wave}}}=\frac{5}{16} \Gamma^{2} c\left(1+c^{2}\right)^{2} .
$$

Figure 9 compares the theoretical and the experimental data, where the theoretical predictions result from evaluating the above formula. The results are in good agreement. It is worth mentioning that the theoretical curve in Figure 9 is based on the theoretical value of $U_{\text {osc }}$, which is less than the experimental value by a factor of 1.1 (see Figure 7). Nevertheless, a good agreement between the theoretical data and the experimental results reveals that the azimuthal steady streaming is generated in the viscous Stokes layer, and the contribution of the Longuet-Higgins mechanism [9] is negligible.

This result gives the opportunity to determine the stability parameters of the centrifugal instability in the viscous boundary layer [5] and the onset of a quasistationary relief at the interface between the granular medium and the fluid inside a rotating cylinder [4]. The specific feature of these problems is the inability to experimentally measure the velocity of the oscillatory fluid motion near the Stokes boundary layer. We found that (10) enables the calculation of the oscillatory component of the velocity using the steady streaming velocity. 


\section{Conclusion}

We have studied the dynamics of a low-viscosity fluid in a rapidly rotating horizontal cylinder. We report the first experimental results on the oscillatory flow of an annular fluid layer under gravity. In the rotating frame, the force of gravity induces the azimuthal fluid oscillations with frequency equal to the velocity of the cylinder rotation. If the free surface is unperturbed and gravity waves are excluded then the velocity amplitude is found to be proportional to the parameter $\Gamma$, which is the ratio of gravitational force and centrifugal force. The experimental data agree well with theoretical predictions. The azimuthal oscillations are regarded as a surface wave propagating opposite to the direction of the cylinder rotation. This wave induces viscous boundary layers near the rigid wall and the free surface and generates the azimuthal steady flow. Considering the impact of boundary layers, we reveal that the steady streaming is generated mainly in the Stokes boundary layer near the rigid wall. According to our measurements, the steady streaming velocity is scaled as $u \sim \Gamma^{2}$.

Therefore, in a uniformly rotating cylinder, liquid performs a combined motion of steady rotation and azimuthal oscillations which is qualitatively similar to the librationdriven flow. The obtained results can be useful to evaluate the stability parameters of the centrifugal instability in the Stokes boundary layer and the onset of ripples at the interface between fluid and granular medium in a rotating or librating cylinder.

\section{Conflict of Interests}

The authors declare that there is no conflict of interests regarding the publication of this paper.

\section{Acknowledgment}

The work is supported by Grant 14-11-00476 of the Russian Scientific Foundation.

\section{References}

[1] M. Nezami, M. M. Mohammadi, and A. Oveisi, "Liquid sloshing in a horizontal circular container with eccentric tube under external excitation," Shock and Vibration, vol. 2014, Article ID 507281, 15 pages, 2014.

[2] G. Seiden and P. J. Thomas, "Complexity, segregation, and pattern formation in rotating-drum flows," Reviews of Modern Physics, vol. 83, no. 4, article 1323, 2011.

[3] O. M. Phillips, "Centrifugal waves," Journal of Fluid Mechanics, vol. 7, pp. 340-352, 1960.

[4] V. Dyakova, V. Kozlov, and D. Polezhaev, "Pattern formation inside a rotating cylinder partially filled with liquid and granular medium," Shock and Vibration, vol. 2014, Article ID 841320, 9 pages, 2014.

[5] V. G. Kozlov and D. A. Polezhaev, "Stability of rimming flow under vibration," Microgravity Science and Technology, vol. 21, no. 1-2, pp. 79-82, 2009.

[6] R. D. Lorenz, B. W. Stiles, R. L. Kirk et al., "Titan's rotation reveals an internal ocean and changing zonal winds," Science, vol. 319, no. 5870, pp. 1649-1651, 2008.
[7] J. L. Margot, S. J. Peale, R. F. Jurgens, M. A. Slade, and I. V. Holin, "Large longitude libration of mercury reveals a molten core," Science, vol. 316, no. 5825, pp. 710-714, 2007.

[8] G. K. Batchelor, An Introduction to Fluid Dynamics, Cambridge University Press, Cambridge, UK, 1967.

[9] M. S. Longuet-Higgins, "Mass transport in water waves," Philosophical Transactions of the Royal Society of London Series A: Mathematical and Physical Sciences, vol. 245, no. 903, pp. 535581, 1953.

[10] A. L. Zuev and K. G. Kostarev, "Certain peculiarities of the solutocapillary convection," Physics-Uspekhi, vol. 51, no. 10, pp. 1027-1045, 2008.

[11] A. Frumkin and V. G. Levich, "On surfactants and interfacial motion," Zhurnal Fizicheskoi Khimii, vol. 21, pp. 1183-1204, 1947.

[12] V. G. Kozlov and N. V. Kozlov, "Vibrational hydrodynamic gyroscope,” Doklady Physics, vol. 52, no. 8, pp. 458-461, 2007.

[13] A. A. Ivanova, V. G. Kozlov, and A. V. Chigrakov, "Dynamics of a fluid in a rotating horizontal cylinder," Fluid Dynamics, vol. 39, no. 4, pp. 594-604, 2004.

[14] V. V. Dyakova and D. A. Polezhaev, “The oscillatory fluid motion inside a rapidly rotating cylinder under gravity," in Proceedings of the 5th International Scientific School of Young Scientists "Waves and Vortices in Complex Media", pp. 35-37, MAKS Press, Moscow, Russia, 2014.

[15] H. P. Greenspan, The Theory of Rotating Fluids, Cambridge University Press, Cambridge, UK, 1968.

[16] D. A. Polezhaev, Experimental investigation of vibrational dynamics of centrifuged liquid layer in a rotating cylinder [Ph.D. thesis], Perm State University, Perm, Russia, 2006 (Russian). 


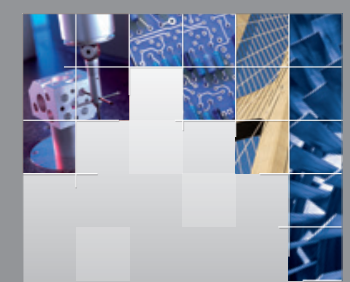

\section{Enfincering}
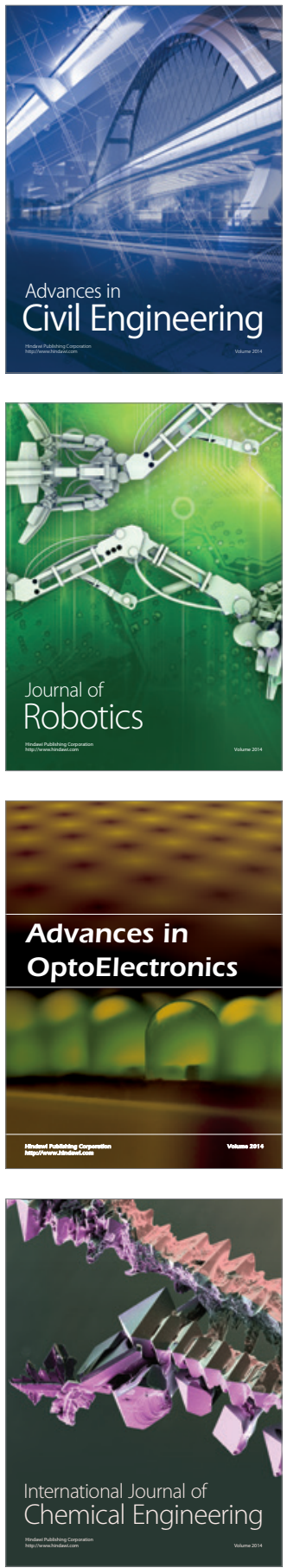

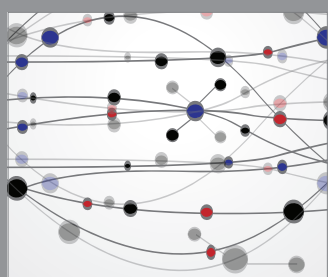

The Scientific World Journal

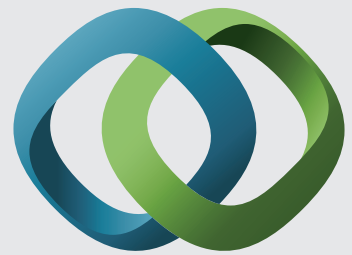

\section{Hindawi}

Submit your manuscripts at

http://www.hindawi.com
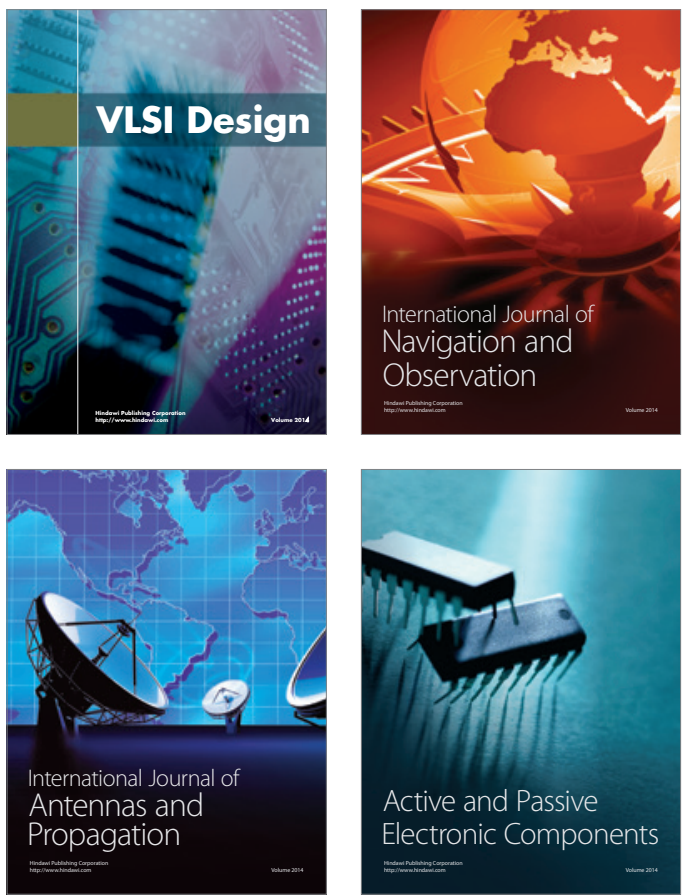
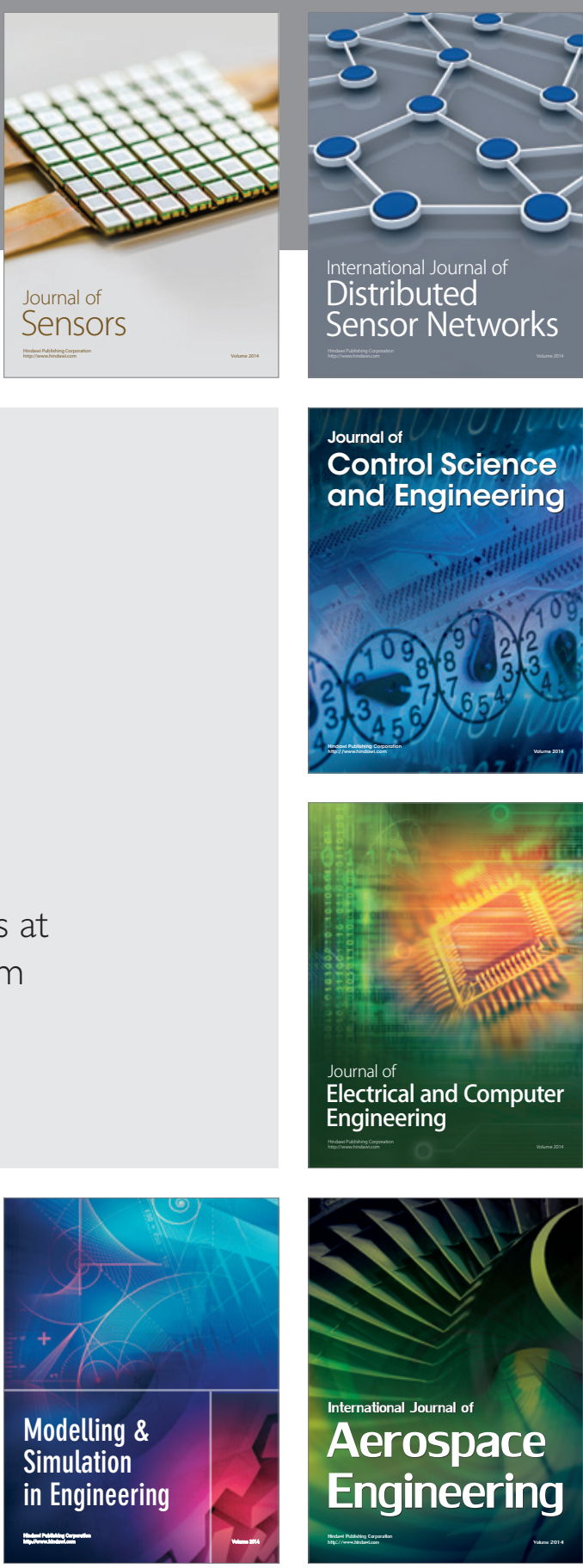

International Journal of

Distributed

Sensor Networks

Journal of

Control Science

and Engineering
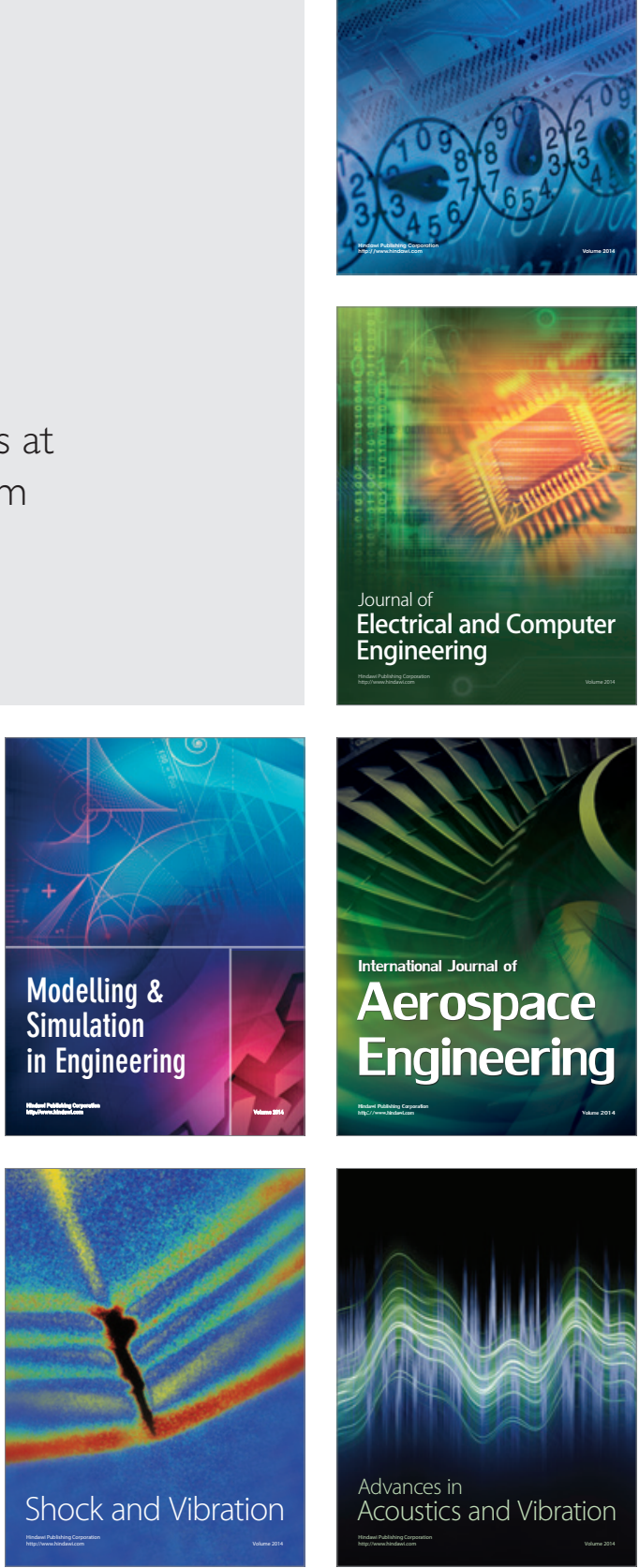